\title{
Nación, identidad y ciudadanía: del ejercicio de inclusión al de exclusión
}

\author{
Danghelly Giovanna Zúñiga Reyes
}

\begin{abstract}
One of the most distinct characteristics of the contemporary world is the complex and often troubled relationship between identity and citizenship. In this article the author presents a framework of analysis, following four authors, in which citizenship could be understood in a double aspect: on the one hand, tied to the belonging to a certain territory from birth, and on the other hand, constructed from the recognition of laws and of rights that are imposed and internalized. In order to come to a conclusion, the article traces the notions of identity and citizenship from the most exclusive notion: from that of the foreigner, which today contains an infinity of theoretical, political and social difficulties.
\end{abstract}

\section{Introducción}

Considero que la distinción entre memoria y olvido puede establecerse en sociedades que han interiorizado claramente períodos de comienzo y final. Así, para los europeos es claro que las guerras mundiales comenzaron en una fecha y se terminaron en otra, que el Holocausto produjo millones de muertos pero que los criminales fueron juzgados, que -especialmente en el caso francés- los períodos de cambio de régimen han tenido un límite temporal claro y distinto y que, de alguna manera, ha podido detenerse el curso equivocado que llevaba la historia. El ejercicio de memoria y olvido requiere estos límites -seguramente simbólicos y arbitrarios, pero límites al fin y al cabo-, requiere esta representación colectiva de una historia que se ha vuelto a encauzar. El caso colombiano parece ser distinto. $\mathrm{Al}$ repasar la historia nacional encontramos que nuestra memoria colectiva no se nutre de fuertes símbolos y mitos fundacionales ni está claramente delimitada por períodos históricos de principio y fin, aunque los historiadores insistan en ello y los establezcan con rigor y precisión. El efecto "eco" todavía no se constituye en mecanismo propiciador del encuentro entre memoria e historia. Por ello se 
hace necesario seguir reflexionando sobre dos ejes: nuestro papel en la corriente general de construcción moderna del Estado-Nación y nuestras particularidades y singularidades que se apartan del "tipo ideal".

La sociología ha explorado, desde Simmel hasta Bauman, una figura estratégica que sirve como prototipo de los fenómenos de identidad: el extranjero. Para Simmel, este es alguien que tiene un doble carácter: no es uno de los nuestros pero nos confronta. El extranjero proviene de un origen diferente y remoto, es un prototípico ejemplo de ambivalencia que significa, según palabras de Bauman, "la posibilidad de asignar más de una categoría a un objeto o evento" (1991). Precisamente porque el extranjero rehúsa pertenecer al "nosotros" se convierte en una constante amenaza al mundo del orden. Bauman señala que el contacto con el extranjero produce dos clases de temor: en primer lugar, la heterofobia, es decir el resentimiento de la diferencia asociado con algo que perturba el orden de lo familiar; en segundo lugar, la proteofobia, es decir, el sentimiento de que ese otro no se ajusta a la estructura del orden establecido. Es por ello que constantemente intentamos "domesticar" al extranjero -y la ambivalencia que él comporta- mediante el uso de estrategias de neutralización y de estigmatización (Bauman, 1995).

El extranjero introduce heterogeneidad y ambivalencia. El extranjero "perturba la confortable tranquilidad de la vida haciendo preguntas que ninguno de los 'locales' recuerda haber hecho o contestado". Tales preguntas convierten en acertijos lo que era auto-evidente, es decir, desfamiliarizan lo familiar (Bauman, 1990). Igualmente, el extranjero nos lleva a pensar que la identidad se define precisamente contra ellos, puesto que al no ser incluidos en un "nosotros" nos permite formular de manera más precisa una definición de ese "nosotros". La teoría social, según este mismo autor, consistiría en seguir al extranjero, en la medida en que la "familiaridad" es "el enemigo de la indagación y de la crítica" y, por lo tanto, la función de la teoría social sería la de actuar como un "entrometido y, a veces, irritante extranjero" (Bauman, 1990). Siguiendo la recomendación de Bauman, tomo esta metáfora para aproximarme a las nociones de nacionalidad, identidad y ciudadanía.

\section{El Estado moderno, grupos étnicos y nación}

El advenimiento de la modernidad en Occidente es concomitante con la definición del Estado-Nación. A partir de allí, la reflexión política se ha hecho mucho más sofisticada y compleja. Nuevos fenómenos como la extensión del capitalismo, la conformación de diversas experiencias democráticas, el surgimiento de partidos políticos de masas y la ampliación del ámbito de acción 
individual y de los derechos individuales, han dado pie a un sinnúmero de teorías y proyectos políticos cuya variedad puede observarse hoy en día. El Estado moderno, definido por Weber como "[...] aquella comunidad humana que en el interior de un determinado territorio reclama para sí (con éxito) el monopolio de la coacción física legítima [...] como fuente única del "derecho" de coacción" (1997: 1056), ha sido el resultado de este proceso. De esta forma se tiene que íntimamente ligada a la concepción de territorio está la de nación, la cual se entremezcla con el Estado dando así paso al Estado-Nación, y la cristalización de esta simbiosis será la nacionalidad.

El Estado-Nación supone, pues, dos grandes planos consolidadores: uno donde sean explícitas las condiciones externas y otro donde sean las condiciones internas las que determinen su construcción. En el plano externo se tiene, en primer lugar, la identidad colectiva ligada a un territorio y a las gestas que se han desarrollado para su defensa. De ahí que sea tan importante para la nación tener una memoria fuerte, representada constantemente para ser recordada por distintas generaciones. Los medios de comunicación juegan un papel preponderante en esta presentación constante de lo que nos mantiene juntos. En este punto se da un primer paso definitorio de nación a partir de la primera distinción de lo "qué no soy" con respecto a otro que será mi eventual vecino de frontera territorial. El segundo paso que se da es el ejercicio del monopolio de la coacción física sobre un territorio, lo cual supone la legitimidad de esta coacción y tiene sustento en la voluntad nacional.

Fue Max Weber el único pensador importante que puso especial cuidado a la distinción entre grupo étnico y nación, aportando así una clave analítica para distinguir entre procesos diferentes y anunciar algunos de los problemas que afronta la sociedad contemporánea. Weber define grupo étnico como:

[...] aquellos grupos humanos que, fundándose en la semejanza del hábito exterior y de las costumbres, o de ambos a la vez, o en recuerdos de colonización y migración, abrigan una creencia subjetiva en una procedencia común, de tal suerte que la creencia es importante para la ampliación de las comunidades (1997: 318).

A su vez, resulta esclarecedor el uso del concepto de nación que este mismo autor desarrolla:

Siempre [...] nos refiere al "poder" político y lo "nacional"-si en general es algo unitario- es un tipo especial de pathos que, en un grupo humano unido por una comunidad de lenguaje, de religión, de costumbres o de destino, se vincula a la idea de una organización política propia, ya existente o a la que aspira y cuanto más se carga el acento sobre la idea 
de “poder", tanto más específico resulta ese sentimiento patético (1997: 327).

Nacionalidad puede ser definida, entonces, como "forma comunitaria de vínculo entre muchos individuos que comparten el 'sentimiento subjetivo de construir un todo' y que identifican tal todo con el origen, la trayectoria, la actualidad y el porvenir de un pueblo en específico" (Hernández, 1991: 38). También, como memoria colectiva o como interés de un grupo de perpetuarse sobre la Tierra. De esta manera podríamos muy brevemente decir que esta doble construcción, la de la etnia y la de la nación, comportan dos miradas en direcciones distintas pero complementarias: la etnia mira al pasado y la nación al futuro.

En una perspectiva complementaria a la presentada por Weber, Talcott Parsons entiende la sociedad moderna como fruto de un complejo institucional desarrollado a partir de cuatro grandes procesos revolucionarios:

- La revolución religiosa, que abrió las compuertas éticas y morales para el desarrollo de las modernas prácticas industriales y comerciales así como para la vinculación estrecha de la ciencia en los procesos productivos y permitió la secularización del Estado y la inserción de diversas creencias personales como parte legítima de la acción individual.

- La revolución industrial que permitió la movilización de los factores productivos y la diferenciación moderna entre las clases.

- La revolución política -de corte democrático- ligada estrechamente al nacionalismo que permitió la acción efectiva del individuo al concebirlo ya no como súbdito sino como ciudadano y que dio pie a la burocracia moderna.

- La revolución educativa -teorizada también como científico-técnicaque alteró la estructura ocupacional derivada de la industrial y dio pie al desarrollo extensivo de la educación superior y al surgimiento de las nuevas profesiones (Parsons 1978).

Como fruto de este cuádruple proceso revolucionario, el desarrollo de la sociedad moderna implicó la absorción de minorías étnicas que, agrupadas bajo el concepto de ciudadanía respaldado por el sistema educativo de carácter universalista, terminarían incluyéndose en la forma del Estado-Nación.

La consecuencia, pues, podría ser claramente esbozada así: a la vez que la burocracia y el mercado se liberan de las formas tempranas de control social y 
moral, ${ }^{1}$ el Estado, como agencia de la nación, afirma su control sobre las formas culturales más generales (el idioma y la religión) a pesar de que estas tiendan a extenderse más allá de sus límites territoriales. No obstante, esta unidad tiene que confrontar diversos conflictos internos que deben ser conciliados y reconciliados a partir de la regulación del mercado y de la competencia política en un sistema representativo que, finalmente, es lo que conforma las instituciones de la democracia.

Los Estados nacionales en sus inicios tuvieron, no obstante, que manejar los conflictos con las minorías etnoculturales bajo su jurisdicción. Las condiciones sociales, políticas y económicas desde las cuales se erigió la modernidad exigieron regularización, uniformidad, y homogeneidad: se abolieron barreras aduaneras, se mejoraron los sistemas de transporte y se consolidó un mercado nacional que fue transformando las economías regionales. Esto fue acompañado de una fuerte tendencia a la uniformidad cultural, a la homogeneización y a la consolidación de un núcleo simbólico común. Así pues, la identidad en la forma de nación, abarca dos procesos diferentes: uno de ellos tiene que ver con las instituciones económicas y políticas y el otro con la vida cotidiana en contextos más domésticos, más próximos al accionar diario de cada individuo. Si bien los dos ámbitos se vuelven relativamente independientes, en tiempos de guerra o de amenaza externa -real o construida a partir de cualquier medio- se puede apreciar un fuerte incremento de la solidaridad y de la identificación moral con el Estado-Nación, lo que a su vez contribuye a que el concepto de ciudadanía posea una intensa carga moral. En este momento la idea de nación, que en su origen fue inclusiva, se puede volver excluyente y agresiva. El individuo puede, incluso, aceptar que su estatuto de ciudadanía sea disminuido o anulado en aras de los deberes colectivos.

La ciudadanía implica membresía, pertenencia a un Estado, es decir, a un sistema político e igualdad sin distinciones ante dicho Estado, mientras que la noción de identidad nacional (o étnica) implica el reconocimiento de lazos comunes que, a su vez, incluyen algún grado de exclusión y segregación. El uno se ubica en el ámbito de la política, el otro en el de la cultura. En situaciones históricas particulares cualquiera de los dos puede crecer en detrimento del otro y, por lo tanto, producir diferentes resultados. Mientras que a la luz de las teorías clásicas de la modernización se asumió la desaparición paulatina de grupos solidarios caracterizados por identidades lingüísticas, religiosas o culturales, el mundo contemporáneo parece presenciar la reafirmación, redescubrimiento o

\footnotetext{
${ }^{1}$ Tal como podría desprenderse de las famosas dicotomías comunidad-asociación (Töennies) y solidaridad mecánica-solidaridad orgánica (Durkheim) que, a su vez, se reflejan en aquellas otras tan usadas por los investigadores sociales: rural-urbano, tradicional-moderno, feudalismo-capitalismo, etc.
} 
reconstrucción de tales identidades como fruto precisamente de la extensión de la economía capitalista, del imperio de los Estados nacionales y del desarrollo de un sistema legal de alcances internacionales, proceso este que ha sido teorizado por diversos analistas como propio de la llamada sociedad postmoderna (Borja, Castells, 1998).

El interés moderno en los problemas de etnicidad o de identidad étnica parece tener dos grandes fuentes. En primer lugar podemos mencionar los cambios históricos acaecidos después de la Segunda Guerra Mundial y a partir de los procesos de descolonización. Lo que las ciencias sociales de principios de siglo consideraron como inevitable, la desaparición de los nacionalismos y de las identidades étnicas debido a la modernización, la industrialización y el individualismo, nunca sucedió cabalmente. Por el contrario, las identidades étnicas y los nacionalismos crecieron en importancia durante la última mitad del siglo. Según datos de la Organización de Naciones Unidas, 35 de 37 conflictos armados en 1991 eran conflictos internos y la gran mayoría de ellos tenían bases étnicas. De otra parte, la entrada de trabajadores inmigrantes y refugiados a Estados Unidos y Europa ha generado el surgimiento de minorías étnicas en dichos territorios que demandan algún tipo de reconocimiento político y de estrategias multiculturales en dichas zonas. Como ejemplo de esto podemos presentar la Declaración de Morelia, ${ }^{2}$ en la cual se hace énfasis en el hecho de que se ha criminalizado la situación de "individuos indocumentados" en los países receptores de migrantes. La exigencia por el reconocimiento de los derechos humanos se está trasladando a la exigencia del reconocimiento de derechos civiles, estrategia que se presenta como propuesta para que disminuyan los impactos negativos que tiene la migración.

En segundo lugar, como lo ha señalado Köenig (1998), el término etnicidad aparece por primera vez en el diccionario Oxford en 1972. Sin embargo, la palabra "étnico" es mucho más antigua ya que proviene de la etnia griega (que a su vez se derivó de ethnikos), cuyo significado original era pagano. Hasta mediados del siglo XIX se utilizó en inglés para denotar características raciales. En los Estados Unidos, alrededor de la Segunda Guerra Mundial se utilizó el término para referirse diplomáticamente a los inmigrantes judíos, italianos e irlandeses. Posteriormente adquirió diversas acepciones hasta llegar a significar "minoría cultural", aunque los enfoques más modernos prefieren utilizarlo para referirse a cualquier tipo de unidad cultural, sea mayoritaria o minoritaria. La creciente preocupación de los científicos sociales por este tema indica un desplazamiento del interés hacia las

\footnotetext{
${ }^{2}$ Declaración de la Primera Cumbre de Comunidades Migrantes Latinoamericanas, realizada del 10 al 13 de mayo de 2007 en Morelia, Michoacán, México. Para mayor información ver: http://www.cumbredemigrantes.org
} 
áreas y problemas de integración y diferenciación cultural. Los Estados Unidos se presentaron, por ejemplo, como el crisol de nacionalidades y etnias. Parecía cumplirse allí el gran ideal de la modernidad. Sin embargo, muchos de esos grupos étnicos mantienen, en su vida comunitaria y en sus costumbres más arraigadas, todavía un fuerte sentimiento de separación y de preservación.

Se deriva pues una compleja relación entre etnicidad y nacionalismo. Parece existir algún acuerdo, siguiendo a Max Weber, en que etnicidad se refiere a grupos que por diversos factores se atribuyen una historia común pero que no necesariamente reclaman una expresión política en la forma de Estado. Nación enfatizaría el polo opuesto, el de destino común y una referencia directa a la asociación política. De allí que un grupo étnico, en circunstancias particulares, puede expresarse como nación al demandar que los límites culturales coincidan con los límites políticos.

\section{Estrategias nacionales frente al problema de las identidades étnicas}

La mayoría de los científicos sociales y pensadores del siglo XIX y de comienzos del XX consideraron que el problema de las "minorías" terminaría por disolverse en el marco del desarrollo económico y tecnológico moderno. Desde Saint-Simon hasta la Tercera Internacional encontramos ese particular énfasis en el carácter unificador y homogeneizante del capitalismo moderno que terminaría por destruir las barreras entre los grupos étnicos y raciales. La fuerza de la industrialización -cualquiera que fuese la forma política que la contuviera- culminaría devaluando las identidades etnoculturales, dando pie a una sociedad no diversificada, donde terminaría por imponerse la ciudadanía universal. El camino hacia la homogeneización fue diverso: en algunos casos las "minorías étnicas" fueron asimiladas de manera voluntaria o forzada, en otros se las consideró no asimilables y el resultado fue migración forzada, segregación, limpieza étnica o genocidio.

En las sociedades contemporáneas se han dado dos grandes acontecimientos que han traído a primer plano los problemas relativos a la etnicidad y a las identidades culturales. De una parte, la descolonización acaecida después de la Segunda Guerra Mundial y, por otra, el derrumbe de los regímenes comunistas. La magnitud de este cambio ha sido de tales dimensiones que basta señalar que el número de miembros de las Naciones Unidas ha pasado de los 50 Estados originales en 1945 a los 192 miembros actuales. El último Estado admitido fue Montenegro (28 de junio de 2006). Hacia 1984, 85 colonias se habían vuelto Estados independientes. La descolonización trajo desafíos similares a los de la 
construcción de los Estados nacionales de Europa y América, pues se trataba -y se trata- no sólo de hacer política y económicamente viables a esos nuevos Estados, sino además de incorporar bajo un mismo marco cultural a los diversos grupos étnicos.

Pero esta realidad toca a todo el espectro de Estados nacionales. Según estudios del Programa Gestión de las Transformaciones Sociales (MOST) de la UNESCO, sólo el 10 o $15 \%$ de los países del mundo pueden considerarse hoy como étnicamente homogéneos (MOST 1995). Los procesos de migración han puesto en la agenda política de muchos estados el tema de la diversidad étnica (Köenig 1998). Esto ocurre debido al incremento de dos factores: la migración laboral y el número de parejas en las cuales uno de los miembros nació en el extranjero.

Las nuevas minorías étnicas inmigrantes tienen su propio sentido de identidad. Esto no debe sin embargo ser pensado en términos de inmutabilidad y límites claramente definidos. De nuevo tropezamos también con diversas respuestas ante esta presencia multicultural de los inmigrantes. De un lado, se puede intentar la asimilación de tales minorías a la categoría de ciudadanos locales. De otro, se puede intentar que asuman una especie de ciudadanía de segunda clase. $\mathrm{O}$, finalmente, se puede reconocer su diversidad cultural en una esfera comunal privada, siempre y cuando los inmigrantes adopten la cultura pública de la sociedad receptora, descartando, obviamente, el caso extremo -que no indica aceptación del migrante- de su exclusión bajo formas extremas de limpieza étnica o mimesis cultural forzada. Hay, por supuesto, dos extremos en los cuales se afirma la etnicidad subordinada. Uno, aquel en el cual se cuestiona incluso el monopolio de la violencia legítima de la etnia dominante como en el caso de los católicos irlandeses o de los vascos. Al otro extremo hallamos aquellos grupos que mantienen su propia cultura étnica en una especie de entidad étnica subalterno-nacional que se conforma con alguna clase de autonomía política.

De todo este complejo proceso de formación y afirmación de identidades culturales puede resultar una oposición al concepto de ciudadanía, por lo menos al que deriva de la Revolución Francesa y que define el estatus de pertenencia política en los modernos Estados nacionales. La legislación en diversos paísesno solamente en aquellos económicamente más avanzados ${ }^{3}$-puede ser un buen ejemplo de esta oposición. De hecho, como fruto de procesos históricos particulares y complejos, muchas constituciones han contemplado para las "minorías" derechos especiales - de carácter político, social y cultural- que no sólo menguan el alcance de la pauta universalista de la ciudadanía sino que a

\footnotetext{
${ }^{3}$ La Constitución colombiana de 1991 puede ser un buen ejemplo.
} 
veces la contradicen abiertamente. ${ }^{4}$ Como ha señalado el sociólogo inglés John Rex (1996) puede decirse que la diferencia entre el viejo nacionalismo y el nuevo consiste en que este conduce a la exclusión, mientras que aquel tenía por meta la inclusión. Los movimientos nacionalistas extremos, tanto en Estados Unidos como en Alemania, Francia e Inglaterra, parecen definir la identidad nacional no con referencia al extranjero sino principalmente al inmigrante, que pasa a ser el extranjero que vive dentro de sus fronteras.

Así pues, podríamos pensar en dos grandes tipos de nacionalismo. El primero, llamado "nacionalismo cívico", se encuentra ligado estrechamente a la noción de ciudadanía en el marco de un territorio donde el Estado-Nación ejerce su soberanía legítima. Su referente es el Estado como comunidad política. El segundo, conocido como "nacionalismo etnohistórico" está ligado a la idea de comunidad cultural histórica que busca diferenciarse de otras comunidades culturales y que no necesariamente se inscribe en los límites de un Estado en particular, aunque teórica y prácticamente podrían coincidir las dos nociones.

\section{Algunas reflexiones sobre el inicio de la nación y la construcción de ciudadanía en Colombia y América Latina}

En Colombia el proceso de construcción del Estado-Nación se remonta a las luchas de independencia, acompañadas por el discurso de las élites criollas que se pretendía organizador de la sociedad. No quiere decir esto que dicho proceso haya sido unívoco y acumulable dentro de la noción histórica del progreso característica del siglo XIX. En este punto König (1984) señala su propuesta de definición de nación para el análisis del período 1810-1819 en Colombia. Para él nación es:

[...] un orden ficticio determinado por una colectividad de hombres, deduciéndose la naturaleza de este conjunto de los criterios o cualidades diferentes, como los definen por ejemplo las élites. Esos criterios determinantes, que sirven para diferenciar, hacen aparecer, al mismo tiempo, lo diferenciado como un valor especial (aspecto normativo), y de este modo proporcionan una orientación. Pueden ser, por ejemplo, criterios étnicos, criterios culturales o criterios de derecho humanos cívicos. Según las diferentes cualidades que obtengan mayor valor en un orden conflictivo de la nación, se forman naciones diferentes que están sujetas a cambios cada vez

\footnotetext{
${ }^{4}$ De hecho, esta parece ser una nueva fuente de conflicto interétnico que no necesariamente adquiere un carácter abierto. Pueden presentarse inclusive conflictos entre las mismas "minorías" como ya se observa, en el caso colombiano, entre "comunidades negras" y "comunidades indígenas".
} 
que los criterios determinantes y su valor o una mezcla de criterios cambien en el transcurso de los tiempos y que sean adaptables a las constelaciones reales del poder en el desarrollo histórico (König 1984: 392).

Los elementos generales y fundamentales que se deben establecer para dar cuenta del tipo de discurso que construye la nación y el Estado-Nación son:

1. Lo que entienden por nación en una determinada situación histórica los personajes decisivos de una sociedad.

2. Los criterios con los que incluso para el pueblo se define la nación.

3. Las formas de legitimación del orden político.

4. La justificación de la fundación del Estado.

Así pues, ¿cuáles son los criterios que usaron los dirigentes políticos de la Nueva Granada para constituir la nueva nación? La nación pasa a ser el sustrato sobre el cual se construyen las relaciones sociales, económicas y políticas. Se le da así un papel activo a esta construcción pues de ella depende la estructuración del orden político. Como lo señala Sábato (1999), esta pregunta sobre la construcción de la ciudadanía política va unida a la pregunta sobre cómo se construyeron las comunidades políticas en América Latina.

En los momentos del inicio de las luchas de independencia se requería una fuerza unificadora frente al enemigo, que es un enemigo común a todos los nacidos en América. Las élites encuentran en lo indígena ese común denominador. Por ello se hace una remembranza de lo aborigen; ejemplo de esto es la recuperación del nombre indígena de regiones como Cundinamarca para la provincia de Santafé de Bogotá o Calamar para Cartagena: “(...) en cuadros y en monedas aparecía la India coronada como símbolo de libertad" (König 1984:395). Se busca también la integración de todos los sectores sociales, por ello se borra momentáneamente el imaginario negativo de lo indígena:

Mientras que antes se había hablado del indio estúpido, primitivo o salvaje, ahora el indio aparecía en los escritos políticos como pobre hombre digno de lástima y de compasión al cual la conquista española le había obstaculizado su desarrollo y quien durante la época colonial había sido descuidado y humillado por la opresión española (König, 1984: 395).

Esto es importante si se tiene en cuenta que desde finales del siglo XVIII Colombia goza de una mayoría de población mestiza, como da cuenta el censo étnico-social de 1788 en el cual la composición en el territorio colombiano aparece así (Silvestre citado por Jaramillo, 1984: 342): 


$\begin{array}{lll}\text { Blancos (españoles y criollos) } & 290.599 & (32.6 \%) \\ \text { Mestizos } & 391.869 & (43 \%) \\ \text { Indígenas } & 151.785 & (17 \%) \\ \text { Esclavos } & 57.077 & (6.4 \%) \\ \text { Total } & 891.330 & (100 \%)\end{array}$

Se olvidó la antigua noción del indio bruto, salvaje para convertirlo en una víctima de la explotación española que, a su vez, representara la situación general de los pobladores. Camilo Torres incluso llegó a argumentar que los criollos eran los vengadores de los indios (König, 1984: 396). De esta manera se comienza a construir, de forma algo forzada, una historia común, una historia integradora. Se inicia así una diferenciación entre los nacidos en América y los que son "extranjeros".

La función simbólica de unificación quedó superada cuando después de la batalla de Boyacá quedó liberada la mayor parte de la Nueva Granada. Entonces ya no era necesaria la construcción de ese enemigo extranjero. Así lo expresó el Congreso de Cúcuta al adoptar como nuevo símbolo de libertad un busto femenino en indumentaria romana (König, 1984: 398). Se pasó entonces a la definición más abstracta de la ciudadanía y el ciudadano, en donde la ciudadanía era la representación de los derechos políticos del ciudadano libre frente a un modelo constitucional. Bolívar le da la visión de igualdad a la definición de ciudadano como la construcción del común denominador de aquellos que son cobijados por el Estado en plena formación. De esta forma los indígenas son sujetos de deberes y derechos en los cuales se hace explícita la necesidad de la propiedad para ser reconocido como ciudadano: estandarte del mundo "civilizado" la propiedad individual actúa en contra de la condición de propiedad comunitaria que se había sostenido en el resguardo indígena. Comienza un nuevo proceso de exclusión por parte de las élites que encuentran en la explotación agrícola su vinculación con el mercado internacional.

Ciudadanía se hizo sinónima de patriotismo. Las luchas de independencia se mantenían parejas al proceso de construcción de Estado en donde los intereses económicos comenzaban a tensionar la cuerda hacia el federalismo y el centralismo. Jaime Jaramillo Uribe exorciza la lectura negativa que se ha mantenido hacia el federalismo argumentando que "[...] el federalismo tenía sus bases reales y en cierto sentido podría decirse que fue una etapa necesaria en la historia de la formación en Colombia" (Jaramillo, 1984: 356). Señala que es un proceso económico, el desestanco del tabaco, el que comienza a darle al Estado a partir de 1850 recursos 
para implementar una administración más adecuada para la consolidación del proceso que llegaría a su fin en 1880 con el ascenso de los conservadores al poder. La Constitución de 1886 hace de Colombia un país con una centralización política y administrativa que va unida a la creación del Ejército Nacional, quitándole así el poder a los caudillos regionales. Sin embargo este proceso va unido de nuevo a la exclusión de los liberales del poder. Se dan los elementos que permiten una ciudadanía formal; los liberales en armas buscan una ciudadanía real en la cual el ejercicio de los derechos humanos y de los del ciudadano sean uno solo.

Al final del siglo XIX se sientan las bases del Estado-Nación en Colombia, pero se inicia el siglo XX con una guerra civil y una fuerte exclusión política,que se mantiene hasta 1930 -a excepción de un pequeño período entre 1904 a 1909- con la presidencia de Rafael Reyes quien trata de hacer por primera vez un gobierno suprapartidista, que realice el sueño de concretar esa ciudadanía. La noción inicial, la de una ciudadanía como privilegio de algunos, se va transformando con el proceso de racionalización del Estado para culminar en una ciudadanía con derechos para todos. La expresión de esta transformación se presenta tanto en los procesos de racionalización política como en los procesos de racionalización administrativa. Podemos decir que la eliminación del sufragio censitario, la participación femenina en la toma de decisiones públicas, la implementación del sufragio universal y la organización de los partidos, fueron procesos que permitieron organizar la participación política de los ciudadanos.

Haciendo un salto cualitativo hasta la Constitución de 1991, con esta se dio en Colombia un paso significativo en el proceso de reconocimiento del otro y en el proceso de integración. No sólo se hizo integración política al permitir y potenciar las denominadas terceras fuerzas, sino también se abrió el camino para la participación de las minorías en los espacios políticos de toma de decisiones. Sin embargo, la actividad política de las terceras fuerzas ha venido disminuyendo, como aseguran algunos autores. y sólo la unión con otros sectores sociales y políticos les permite la sobrevivencia en el ámbito político.

Manuel Antonio Garretón (1996) ha descrito cuatro grandes procesos que determinan los desafíos de las sociedades latinoamericanas a las puertas del siglo XXI:

- construcción democrática

- redefinición del modelo de desarrollo e inserción internacional 
- integración o democratización social

- $\quad$ búsqueda de la modernidad

Si bien esta clasificación es un poco general y vaga, sirve de guía para identificar los ejes temáticos que parecen estar al orden del día en las ciencias sociales de nuestro continente.

La necesidad de la construcción democrática parece derivarse de la vieja idea de que nuestras sociedades fueron definidas -o por lo menos, idealmente se ha procurado que sea así- desde la esfera del Estado. Una sociedad débil habría requerido históricamente la existencia de un Estado fuertemente regulador que, no obstante, por procesos particulares, termina distorsionándose en la forma de hiperburocratización. ${ }^{5}$

La redefinición del modelo de desarrollo implica una más intensa discusión sobre los efectos del neoliberalismo convertido en discurso oficial. En este caso, el tema introducido por Bourdieu sobre la desaparición discursiva de los grandes colectivos -nación, organizaciones sindicales y sociales- y la amenaza política contra su existencia, estaría signando un tema de disputa política, social y cultural. La inserción internacional implica, por otra parte, entender que la globalización significa hoy en día que pasamos de un mundo básicamente geo-político a un mundo primordialmente geo-económico y, sobre todo, geo-cultural.

La integración y la profundización democrática apuntan a los desafíos que plantea la extensión del concepto de ciudadanía, cuya constitución no depende ya exclusivamente de la reivindicación de derechos frente al poder estatal, sino que cubre áreas tan diversas como los medios de comunicación, las relaciones de género, la educación y la ciencia, el medio ambiente, las regiones y localidades. El problema se tornaría más complejo si se considera que quizá solamente pueden identificarse tres grandes procesos históricos latinoamericanos que ayudaron a despertar el espíritu igualitario de las amplias masas desprotegidas: la revolución mexicana, la revolución cubana y el peronismo argentino, procesos que permitieron que, quizás principalmente en el plano formal, se expresara la igualdad entre todos los miembros del Estado.

Por último, el tema de la modernidad ha concitado ya innumerables debates entre los científicos sociales latinoamericanos. Quizá todavía sea útil distinguir entre modernidad y modernización para resaltar que, sin culminar una fase, América Latina ha entrado en un mundo globalizado -aunque cada país lo haya hecho por puertas diferentes. La modernización inconclusa no ha permitido

\footnotetext{
${ }^{5}$ No se me escapa el hecho de que algunos analistas hayan propuesto igualmente una inversión de los términos resaltando la fortaleza y riqueza de la sociedad civil frente a la presencia de un Estado débil.
} 
que el cuádruple proceso revolucionario sugerido por Parsons se complete cabalmente, y por lo tanto se han quedado sin resolver problemas agudos de desarrollo económico y tecnológico y de conformación cultural y política. Si en las décadas de 1960 y 1970 el desafío latinoamericano parecía estar centrado en el desarrollo de burguesías locales capaces de liderar procesos modernos de acumulación de capital -lo que llevó a experimentos distintos, desde el proteccionismo estatal hasta la abierta suplantación de dichas burguesías por capas tecnocráticas o militares "modernizantes"-, hoy la democratización social parece estar ligada al proyecto, a veces confuso y entrabado, de la participación ciudadana. En este caso también el énfasis se ha trastocado con el tiempo. En un principio, participar significaba incorporación, integración, inclusión, pues dicho concepto, convertido en ideario político, estaba ligado a la conformación de naciones independientes y "descolonizadas". Hoy en día los desafíos parecen ampliar dicho concepto para referirlo no solamente al acceso a bienes colectivos sino también a la formación de ciudadanos, es decir, de sujetos capaces de tomar en sus manos las decisiones que afectan todas las áreas de su actividad cotidiana: educación, salud, trabajo, información.

\section{Conclusiones}

Finalmente, para resumir este ya largo recorrido por los temas y problemas de la identidad, la ciudadanía y la democracia, convendría precisar, siguiendo a Etienne Tassin (1997), los siguientes aspectos:

1. Ciudadanía no es nacionalidad: aquella tiene que ver con derechos y se remite a la esfera de la política; esta, en cambio, se relaciona con identidad y se remite al ámbito de la cultura. Nacionalidad es un modo de ser y ciudadanía un modo de actuar que no necesariamente tienen que coincidir. Ambas comportan desafíos diferentes, pues la nacionalidad tiene que ver con la forma de relación con un "otro" que se define como diferente, mientras que la ciudadanía lleva a la necesidad de establecer un espacio público de acción conjunta. Ambos campos pueden coexistir, confundirse, sobreponerse o repelerse según el caso, pero analíticamente es preciso diferenciar los procesos que los constituyen.

2. El ejercicio de la ciudadanía hoy encuentra cuatro grandes temas alrededor de los cuales se vuelve compleja y problemática su definición: primero, el de la legitimidad del orden político y social; segundo, el de la igualdad social; tercero, el del respeto a las diferencias culturales y, finalmente, el de las adhesiones locales. 
3. El éxito de la democracia moderna implica afrontar el desafío de combinar la acción política igualitaria con la diversidad de estilos de vida, identidades étnicas y aun perspectivas plurinacionales. Su reto más grave consiste en darle juego a formulaciones diversas de identidad y ciudadanía.

Así, el problema de la ciudadanía deja de ser un problema de "pertenecer desde el nacimiento" para convertirse en el problema del reconocimiento de leyes que le son impuestas pero con los derechos que de ellas se derivan. La ciudadanía queda enmarcada en la racionalización del ámbito político democrático, en donde las prácticas que son promovidas a su interior no tienen reglas de juego ocultas y son conocidas por todos los participantes en condiciones de igualdad y de inclusión. La identidad entonces no sería un problema de reconocimiento de otros a la pertenencia al grupo por compartir elementos culturales. Sería la exteriorización de la práctica política en un ambiente democrático, en donde los ciudadanos sean capaces de gobernarse a sí mismos sin excluir a nadie que esté regido por las normas que los rigen a ellos.

El desafío es doble, pues, en primer lugar, entender los problemas de cohesión política y de dinámicas culturales exige a la ciencia social al menos un cambio de énfasis: del Estado a la sociedad, de la nación a la complejidad cultural, de la ciudadanía como inclusión a los espacios políticos diferenciados por diversas formas de acción y participación. Y en segundo lugar, las agencias políticas tendrán que pensar la vida democrática de maneras más imaginativas para permitir que la acción del Estado sea la de un facilitador para el despliegue de la vida social en todas sus dimensiones. Infortunadamente, al menos en Colombia, todavía parece prevalecer la tendencia a la unidad y la cohesión por la vía de la coacción física, la homogeneización y el unanimismo.

\section{Bibliografía}

- Bauman, Zygmunt, 1995, Life in Fragments. Essays in Postmodern Morality, Oxford: Blackwell. 1991, Modernity and Ambivalence, Cambridge: Polity

Press. 1990, Thinking Sociologically, Cambridge: Blackwell.

- Borja, Jordi y Manuel Castells, 1998, Local y global. La gestión de las ciudades en la era de la información, España: Taurus. 
- Garretón, Manuel Antonio, 1996, "Democratización, desarrollo, modernidad. ¿Nuevas dimensiones del análisis social?”, en: Excerpta, No. 2, abril 1996.

- Hernández, Miguel Ángel, 1991, "La modernización social y el mundo moderno", en: Gutiérrez, Carlos (ed.), La investigación en Colombia, en las artes, las bumanidades y las ciencias sociales, Misión de Ciencia y Tecnología, Bogotá: Uniandes.

- Jaramillo Uribe, Jaime, 1984, "Nación y región en los orígenes del Estado nacional en Colombia”, en: VV.AA., Problemas de la formación del Estado y de la nación en Hispanoamérica, Bonn: Inter. Nationes.

- König, Hans-Joachim, 1984, "Símbolos nacionales y retórica política en la independencia: El caso de la Nueva Granada”, en: VV.AA., Problemas de la formación del Estado y de la nación en Hispanoamérica, Bonn: Inter. Nationes.

- Koenig, Matthias, 1998, "Democratic governance in multicultural societies. Social conditions for the implementation of international human rights through multicultural policies". UNESCO-MOST [Management of Social Transformations]. http://unesdoc.unesco.org/images/0011/001149/114956eo. pdf.

- Parsons, Talcott. 1978, Autobiografía intelectual, Bogotá: Tercer Mundo.

- Rex, John, 1996, "National Identity in the Democratic Multi-Cultural State", en: Sociological Research Online, vol. 1, no. 2. http://www.socresonline.org. $\mathrm{uk} / 1 / 2 / 1 . h \mathrm{tml}$.

- Sábato, Hilda, (coord.), 1999, Ciudadanía política y formación de las naciones. Perspectivas históricas de América Latina, México: El Colegio de México - Fideicomiso Historia de las Américas - FCE.

- Tassin, Etienne, 1997, Citoyenneté et espace public européens, Institut du management d'EDF et de GDF.

- UNESCO. Programa Gestión de las Transformaciones Sociales (MOST). Boletín de MOST No. 3 junio de 1995, disponible en: www.unesco.org/most/ newlet3s.htm

- Weber, Max, 1997, Economía y Sociedad, Colombia: Fondo de Cultura Económica. 